\title{
Enlighten-GAN for Super Resolution Reconstruction in Mid-Resolution Remote Sensing Images
}

\author{
Yuanfu Gong ${ }^{1,2}$, Puyun Liao ${ }^{1}$, Xiaodong Zhang ${ }^{1, *}$, Lifei Zhang ${ }^{1}$, Guanzhou Chen ${ }^{1}{ }^{\circledR}$, Kun Zhu ${ }^{1}{ }^{\circledR}$, \\ Xiaoliang Tan ${ }^{1}$ and Zhiyong Lv $^{3}$
}

1 State Key Laboratory of Information Engineering in Surveying, Mapping and Remote Sensing (LIESMARS), No.129 Luoyu Road, Wuhan 430079, China; gongsir@whu.edu.cn (Y.G.); LiaoPuyun@whu.edu.cn (P.L.); lifeizhang@whu.edu.cn (L.Z.); cgz@whu.edu.cn (G.C.); zkun@whu.edu.cn (K.Z.); xl_tan@whu.edu.cn (X.T.)

2 Hubei Institute of Land Surveying and Mapping, No.199 Macau Road, Wuhan 430034, China

3 School of Computer and Engineering, Xi'An University of Technology, No.5 Jin Hua South Road, Xi'an 710048, China; zhiyongLyu@xaut.edu.com

* Correspondence: zxdlmars@whu.edu.cn

Citation: Gong, Y.; Liao, P.; Zhang, X.; Zhang, L.; Chen, G.;

Zhu, K.; Tan, X.; Lv, Z.

Enlighten-GAN for Super Resolution Reconstruction in Mid-Resolution Remote Sensing Images.. Remote Sens. 2021, 13, 1104. https://doi.org/ $10.3390 /$ rs13061104

Academic Editor: Edoardo Pasolli

Received: 2 February 2021

Accepted: 9 March 2021

Published: 14 March 2021

Publisher's Note: MDPI stays neutral with regard to jurisdictional claims in published maps and institutional affiliations.

Copyright: (c) 2021 by the authors. Licensee MDPI, Basel, Switzerland. This article is an open access article distributed under the terms and conditions of the Creative Commons Attribution (CC BY) license (https:// creativecommons.org/licenses/by/ $4.0 /)$.

\begin{abstract}
Previously, generative adversarial networks (GAN) have been widely applied on super resolution reconstruction (SRR) methods, which turn low-resolution (LR) images into high-resolution (HR) ones. However, as these methods recover high frequency information with what they observed from the other images, they tend to produce artifacts when processing unfamiliar images. Optical satellite remote sensing images are of a far more complicated scene than natural images. Therefore, applying the previous networks on remote sensing images, especially mid-resolution ones, leads to unstable convergence and thus unpleasing artifacts. In this paper, we propose Enlighten-GAN for SRR tasks on large-size optical mid-resolution remote sensing images. Specifically, we design the enlighten blocks to induce network converging to a reliable point, and bring the Self-Supervised Hierarchical Perceptual Loss to attain performance improvement overpassing the other loss functions. Furthermore, limited by memory, large-scale images need to be cropped into patches to get through the network separately. To merge the reconstructed patches into a whole, we employ the internal inconsistency loss and cropping-and-clipping strategy, to avoid the seam line. Experiment results certify that Enlighten-GAN outperforms the state-of-the-art methods in terms of gradient similarity metric (GSM) on mid-resolution Sentinel-2 remote sensing images.
\end{abstract}

Keywords: super resolution reconstruction; mid-resolution remote sensing images; generative adversarial network

\section{Introduction}

Optical satellite remote sensing images have been applied on varied applications. To better exploit these images and improve image resolution, researchers have turned their attention to super resolution reconstruction (SRR) methods, which turn low-resolution (LR) images into high-resolution (HR) ones. To simplify expression and clarify the distinction from HR images, the results predicted by SRR methods from LR images are called super resolution (SR) images.

In that SRR task exists an ill-posed problem [1]: the methods for this task must spare no effort to exploit prior knowledge. Some methods take prior knowledge by establishing degradation models [2], some others are based on sparse representation and learn a dictionary for transforming between LR patches and HR patches [3,4]. However, the lack of learning ability limits the performance of these methods. Therefore, Dong et al. introduced a convolutional neural network $(\mathrm{CNN})$ of high learning ability into SRR tasks and proposes the SRCNN [5]. However, this method adopts pixel loss to optimize network, and leads to overly smooth results as pixel loss does not take perceptual quality into account, as shown in the first column of Figure 1. Despite the usage of residual learning [6-8], 
recursive learning [9], dense connection [10,11], attention mechanism [8,12], and deep learning architectures to boost performance, the results from pixel-loss-based methods still suffer from the aforementioned issue. Thus, Johnson et al. adopted the perceptual loss to measure semantic similarity [13]. Sajjadi et al. adopted texture loss focusing on gradient similarity defined with Gram matrix [14]. Their results surpass the previous ones, but are still of obvious margin compared with real HR images.

Generative adversarial networks (GANs) have been proved to be effective for generating realistic images thanks to the adversarial loss. Ledig et al. first utilized GAN for SRR task and designed the SRGAN [1,15]. Subsequently, ESRGAN improved it with Residual-inResidual Dense Block (RRDB), relativistic discriminative loss, and perceptual loss without activation [16]. The results from GAN-based methods are realistic and satisfying applied on natural images.
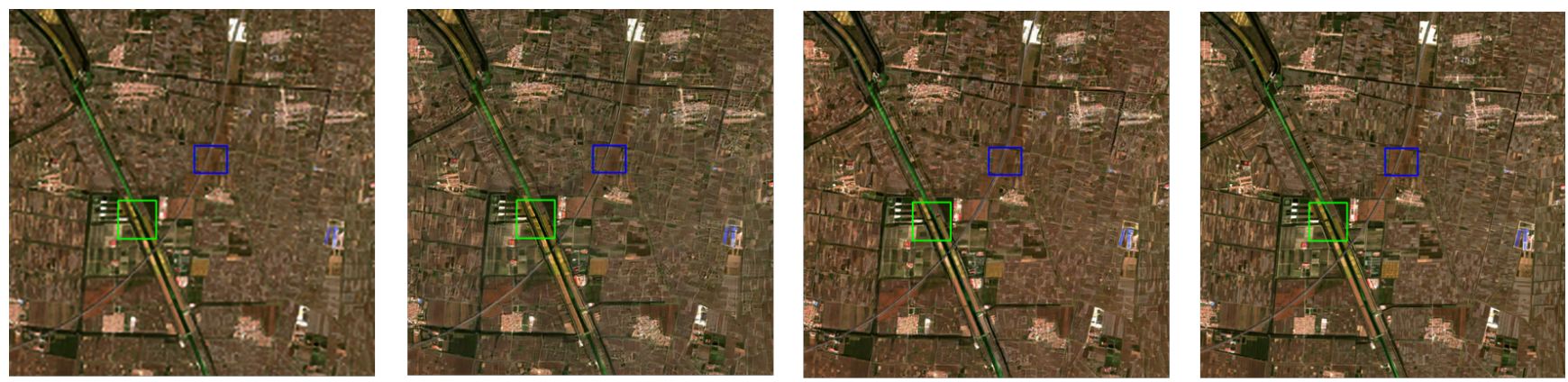

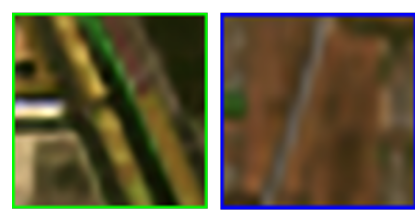

SRCNN

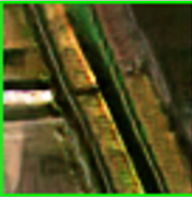

ESRGAN
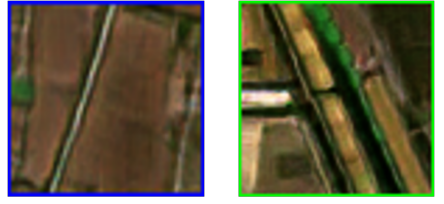

Enlighten-GAN

(Our Proposed)
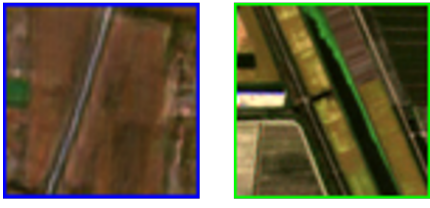

Ground Truth

Figure 1. The super resolution reconstruction (SRR) results of SRCNN, ESRGAN, and our proposed Enlighten-GAN along with the ground truth. We crop and zoom the classic area, in relation to the observation of details. The result of SRCNN, as a representation for pixel-loss-based methods, is blurred for their conservative strategy, while the ESRGAN one is of the unpleasing artifacts. Our result is clear and reliable as a comparison.

Remote sensing images have huge viewport and variable scene, posing challenges to SRR tasks. As shown in the left patch in the second column of Figure 1, ESRGAN produces artifacts such as the unpleasing yellow line at the right end of the field in the left patch and the white road in the right patch, which is actually gray. Variable scenes and complicated environment in mid-resolution remote sensing images exceedingly affect their judgment. Orienting to remote sensing images, Lei et al. proposed the LGCNet, with a skip connection structure similar to Kim et al.'s work [17]. Moreover, Jiang et al. designed the EEGAN to purify the high-frequency map and suppress image noise [18]. Nevertheless, these networks are trained and tested on sub-meter resolution images, which is of clear object contour. Currently, there is no SRR method designed for mid-resolution images.

There is another distinctive issue in remote sensing images in that remote sensing images are highly variable, while the networks can only process small size images limited by memory. In most deep learning applications, the image is cropped into patches and processed separately. Afterwards, these patches are merged into a whole. However, a casual merging strategy leaves a seam line between adjacent patches in the predicted images.

According to the aforementioned issues, in this paper, we propose an SRR method called Enlighten-GAN that primarily focuses on mid-resolution remote sensing images. 
The Enlighten-GAN struggles to induce network converging to a stable and reliable point by varied means. Our main contributions are listed below.

- We design a novel Enlighten-GAN with an enlighten block. The enlighten block benefits the network by setting an easier target to ensure it receives effective gradient. Owing to the varied scale reconstructed results, the enlighten block gains even higher generalization ability. Our proposed Enlighten-GAN proves itself in our comparison experiments on Sentinel-2 images, exceeding the state-of-the-art methods.

- We introduce and employ a Self-Supervised Hierarchical Perceptual Loss for training rather than the conventional perceptual loss defined with VGGNet [15], which is more suitable for SRR-like tasks. We conduct ablation experiment to verify its effectiveness.

- To address the merging issue, we propose a clipping-and-merging method with a learning-based batch internal inconsistency loss, by which the seam lines in the predicted large-scale remote sensing images are dismissed.

The remainder of our paper is organized as follows. In Section 2, we overview the previous SRR methods and GAN variants, for better demonstrating the idea of our paper. In Section 3, we introduce how our proposed Enlighten-GAN works and improves performance. Section 4 validates the utility of our works, followed by Section 5 which concludes our study.

\section{Related Work}

The enlighten GAN is designed for SRR task on mid-resolution optical remote sensing images and applies GAN [19] so as to produce realistic images. In this section, we discuss the related work inspiring our method.

\subsection{Generative Adversarial Network}

GAN consists of a generative model, also known as the $G$, to synthesize fake images, and a discriminative model, abbreviated as the $\mathrm{D}$, to determine whether a given image is synthesized, which improve each other as adversaries [19]. With the D determining whether an image is synthesized or real, the G produces deceptive and thus realistic images. The SRR application of GAN is shown in Figure 2. However, by the cause of non-saturating adversaries between G and D, GAN may be stuck in mode collapse, namely, the phenomenon that the $G$ tend to produce repeated but secure images, forming clustered fake data distributions [20].

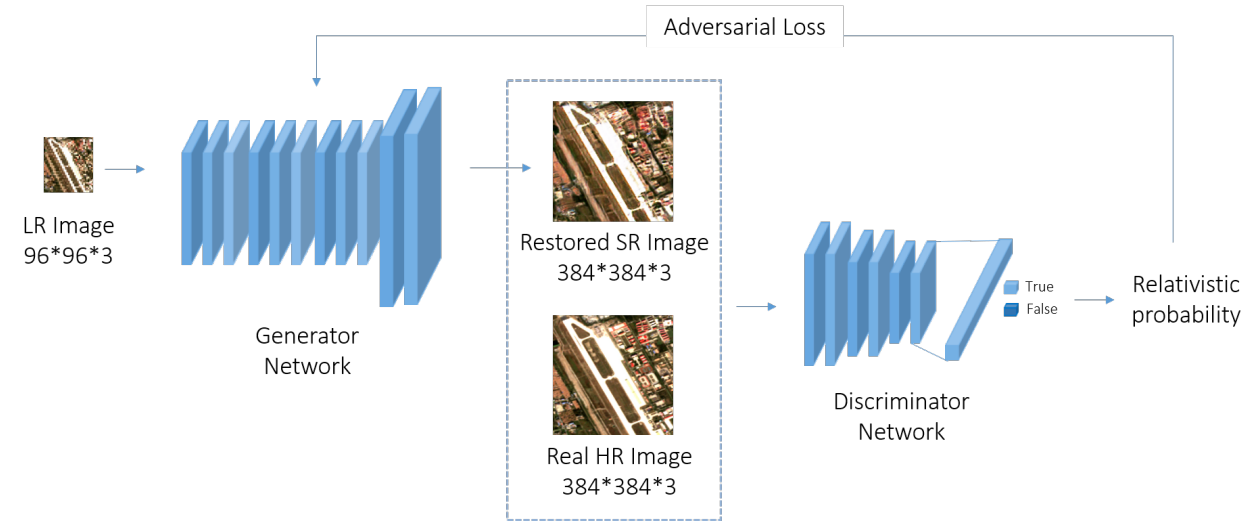

Figure 2. The demonstration of our GAN Structure. Given an LR image, we synthesis SR results from the $\mathrm{G}$ and compare it with the real HR one. The $\mathrm{D}$ is responsible for distinguishing the fake and real one, and thus provides adversarial loss for the training of the $G$.

A variety of attempts have been applied to remove this issue. Mirza and Osindero proposed introducing additional prior knowledge to constrain the data distribution and ease mode collapse; their method uses conditional GANs [21]. Nowozin et al. suggest replacing Kullback-Leibler divergence with a different f-divergence function when mea- 
suring the difference between distributions of real data and fake data [22]. They designed $\mathrm{f}-\mathrm{GAN}$ with varied f-divergence and thus attained varied fake data distributions. SNGAN is developed by employing spectral normalization after each layer. Thereby, the nets follow 1-Lipschitz continuity and thus avoid the vanishing gradient issue [23]. The RaGAN method has been borrowed in ESRGAN, in which the D predict a relative possibility rather than a definitive decision [24]. Furthermore, Wasserstein GAN (WGAN) is proposed by introducing integral probability metrics (IPM) modules to make network models analogous to divergence minimization, as the real data contributes to gradient in IPM-based GAN [20]. While the conventional GAN outputs a sigmoid-activated possibility of an image being true in the D, WGAN and its followings predict a non-activation value called 1-Lipschitz metrics $f_{\omega}$. The positive real sample can be inferred from a high 1-Lipschitz metrics. The 1-Lipschitz metrics distance between the positive sample and negative sample is called the Wasserstein Distance. They further utilized gradient clipping or gradient penalty to constrain thorough modification of weight parameters in one batch, and it performs well in GANs. To demonstrate the application of GAN on SRR tasks, we further introduce the previous CNN and GAN-based SRR methods in the next subsection.

\subsection{Super Resolution Reconstruction}

The super resolution reconstruction problem raises significant attention especially in the remote sensing area in need of improving accuracy. Since Dong et al. introduced deep convolutional neural network and proposed the SRCNN, learning-based methods have been widely applied on SRR tasks, outperforming conventional methods. In order to further boost performance, some took advantages of the sophisticated structure of CNN, while the others introduced enhancement according to the property of SRR task. Specifically, Dong et al. supposed upsampling images after extracting the feature rather than before it to gain efficiency [25]. Inspired by recursive learning, Tai et al. supposed that the network can attain fine SR images by recursively recovering image details from bicubic upsampled LR images [9]. The authors of [26] developed the EDSR by removing batch normalization (BN) layers from residual block. This proves that the BN-free networks perform better due to retaining range flexibility. LapSRN focuses on high-frequency information extracted from images by Laplacian transformation, while the high-frequency information is exactly what the SRR task is concerned with [27]. Furthermore, Shocher et al. believe that the HR details of natural images can be internally implied from itself and proposed a Self-Supervised method named ZSSR, with no need for external image information [28].

To create more realistic images, Ledig et al. first applied GAN into SRR task for its generating ability and designed the SRGAN. To obtain better visual quality, Wang et al. follow the idea of feature map sharing and employ the Residual-in-Residual Dense Block (RRDB), establishing the ESRGAN with relativistic adversarial loss and non-activated perceptual loss. As a matter of fact, GAN is widely applied on optical remote sensing images SRR task, too. Jiang et al. further exploited feature map sharing and proposed ultradense residual blocks and a multi-scale purification unit, constituting the deep distillation recursive network [29]. Afterwards, they found the noise from remote sensing images seriously affects results, and thus designed the EEGAN, which decomposes SRR result to high- and low-frequency part then denoises the high one [18]. Nevertheless, when we apply these methods on mid-resolution remote sensing images, it turns out to be an artifact phenomenon.

The G trained on pixel loss comes lacks high-frequency content especially on remote sensing images. To avoid this problem, most GAN-based SRR methods employ perceptual loss [13], also known as style loss, as addition to pixel loss, so as to attain better generative performance. The perceptual loss measures the diverse between feature maps obtained from two images in a certain layer of deep learning networks. As these feature maps contain more semantic information, it helps the $G$ conduct better adversarial training against the D. Previously, most methods chose VGGNet [15] pretrained on the ImageNet classification dataset [30] to predict feature map for perceptual loss. As classification networks do 
not attach equal attention in each pixel and focus more on the semantic information, we suppose it may not describe the high-frequency information properly. Furthermore, the pooling layers in VGGNet dismiss the HR diverse between images, which is exactly what SRR methods focus on. Therefore, we proposed Self-Supervised Hierarchical Perceptual loss to avoid this hidden trouble, which is discussed in the following sections.

\section{Methodology}

The SRR methods aim to turn a $\mathrm{H} \times \mathrm{W}$ pixel image into $\mathrm{sH} \times s \mathrm{~W}$, where $s$ refers to the upsampling scale. We set $s$ to 4 like most methods do $[1,5,16]$ to ensure the fairness of the performance comparison. Due to the limitation of memory, we crop the given images with overlaps between patches, predict the SR outputs with our GAN network, and eventually merge them carefully at original position into a whole. To take a closer look at our method, we further discuss our network architecture, loss function, and image merging strategy in the followings.

\subsection{Enlighten-Gan}

ESRGAN outperforms the other SRR methods when applied on natural images [16]. Therefore, we take it as our baseline network when designing the Enlighten-GAN. We modified it with the enlighten block and the 1-Lipschitz metrics for stable results in the remote sensing images SRR task. The proposed Enlighten GAN consists of a generative model as shown in Figure 3 and a discriminative model as shown in Figure 4.

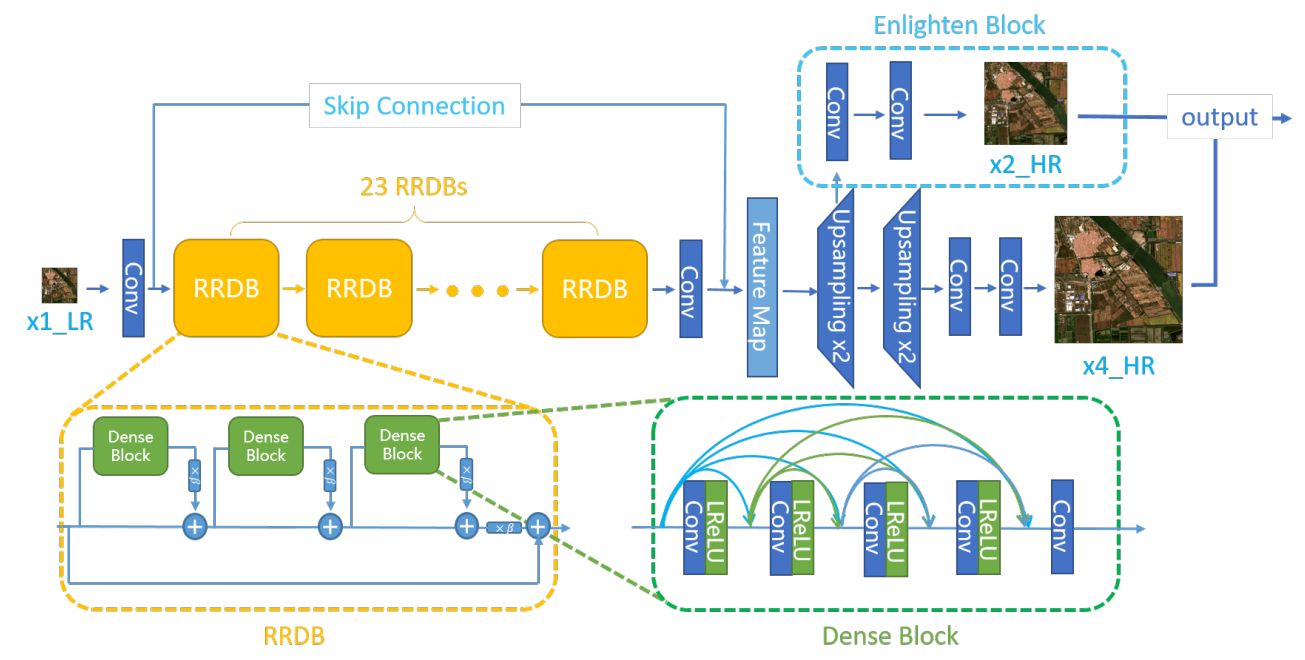

Figure 3. The architecture of the G. The bottom of network extracts feature maps by recursive learning and residual learning, while the top exploits these feature maps to predict multi-level HR images. The "Conv" refers to a convolutional layer with a $3 \times 3$ size kernels, while the RRDB means Residual-in-Residual Dense Block for short. The $\beta$ in RRDB is the residual scaling parameter, which is set as 0.2 .

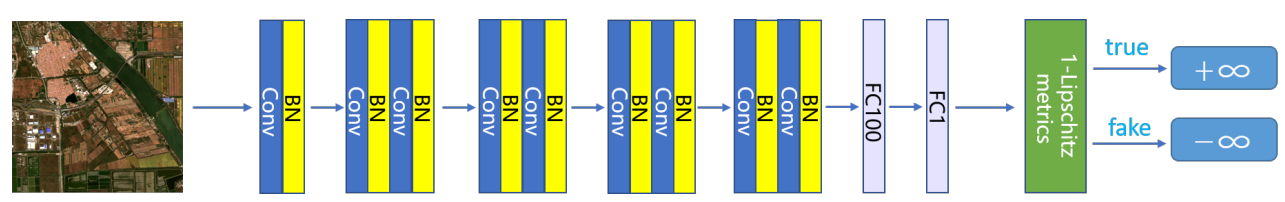

Figure 4. The architecture of the D. It is responsible for encouraging the $\mathrm{G}$ to generate images similar enough to real-world HR data. "BN" is batch normalization for short, "Conv" refers to convolutional layer, and "FC\{N\}" represents a fully connected layers outputting $N$-element array.

The generative model adopts an LR image as input and attains both a 2-times and 4-times HR image as output. After one convolutional layer, 23 basic units named Residualin-Residual Dense Block (RRDB) are arranged to recursively learn the detail from images. 
Each RRDB contains three dense blocks with densely skipping connections and no batch normalization. Subsequently, a skip connection extracts the features from high- and lowlevel layers into a feature map, based on the idea of residual learning [7]. So far, we employed a similar structure to the ESRGAN to extract high-dimension feature maps. We apply this feature map to predict SR images by nearest neighbor interpolation and convolution operation. Besides 4-times outputs, we propose the enlighten block to produce 2-times upsampling results as an easier target. This block enables the feature maps obtained from skip connection to receive a meaningful gradient and learn high-frequency information at an easier and a harder mode alternatively. It prioritizes the network to have more generalization ability owing to its multi-output structure. Thus, the generated HR images from the $\mathrm{G}$ are realistic and natural.

The discriminative model distinguishes the image between true or fake, provides the adversarial loss for generative network, and thus promotes the quality of generated images. The architecture of the $\mathrm{D}$ is brief yet effective. Both synthesized images from the $\mathrm{G}$ and real-world images are fed into this network. Inspired by VGGNet, the pipeline involves sequential convolutional and batch normalization layers, ending with a fully connected layer to predict the possibility of the given image being true. To pursue a stable convergence, we adopted non-activated 1-Lipschitz metrics $f_{\omega}$ as output rather than directly predicting possibility, inspired by WGAN [20]. This modification guides the real-world sample to contribute gradient to our networks, and thus attains a better performance. Notably, when calculating adversarial loss, we focus on the optimization of the 4-times result rather than both results, so that only one discriminative network needs to be trained. The more details of architecture are shown in Figure 4.

\subsection{Model Optimization}

To optimize our designed models, we collected sets of mid-resolution remote sensing images, and downsampled them by 4 times to obtain the LR and HR image pairs as training and validation datasets. The loss function for optimizing our network consists of generative and discriminative loss.

As there are two SR images as result, denoted as $I_{s r \times 2}$ and $I_{s r \times 4}$, we should separately optimize them, and thus form our generative loss function as follows:

$$
\begin{aligned}
\operatorname{Loss}_{G}= & \theta\left(\operatorname{Loss}_{\text {pixel }}\left(I_{s r \times 2}, I_{h r}\right)+\lambda \operatorname{Loss}_{\text {perc }}\left(I_{s r \times 2}, I_{h r}\right)\right) \\
& +\operatorname{Loss}_{\text {pixel }}\left(I_{s r \times 4}, I_{h r}\right)+\lambda \operatorname{Loss}_{\text {perc }}\left(I_{s r \times 4}, I_{h r}\right) \\
& -\alpha \text { Loss }_{\text {adver }}
\end{aligned}
$$

where Loss pixel and Loss perc represent pixel loss and perceptual loss, respectively. The pixel loss is defined as the L2-distance between the ground truth and fake images, while perceptual loss parameterized by $\lambda$ refers to the distance calculated by feature maps of them. Though some found that the L2-distance pixel loss tends to neglect slight differences and thus lead networks to produce blurred yet safe results in CNN networks [26], we observed that it performed well in the GAN structure with the supplement of adversarial loss and perceptual loss. Notably, the loss for the 2-times output part is parameterized by $\theta$ to balance the weight between multi-outputs. Our generative loss function ends with the adversarial loss, Loss $_{\text {adver }}$ parameterized by $\alpha$. It refers to 1-Lipschitz metrics $f_{\omega}$ predicted by the $\mathrm{D}$ and encourages the $\mathrm{G}$ to produce more misleading thus better results. Experiments show the Wasserstein loss predicted by 1-Lipschitz metrics conducts a stable training procedure.

As seen from Equation (1), the perceptual loss item requires effective feature maps to describe the semantic information of images. Previous methods adopted outputs of the layer before fully connected layer in the VGGNet pretrained on ImageNet [16]. However, when validating this idea on remote sensing images, we found networks trained without perceptual loss get a better performance, unexpectedly. We attribute it to applying a classification model to predict feature maps on SRR task. As matter of a fact, classification 
models tend to attach attention to high-level features rather than pixel-level details. Further, the pooling layers in the VGGNet discard high-frequency information the SRR methods recovered, which is exactly the divergence between SR images and HR images. These factors affect the effectiveness of predicted feature maps. Thus, we proposed the SelfSupervised Hierarchical Perceptual Loss, which employs an autoencoder network instead of a classification model to predict feature maps. The autoencoder aims to recover itself from feature maps [31]. Theoretically, the predicted feature maps retain all details from images in order to recover themselves, and thus can be implied to be more reliable.

We build and train a novel and brief autoencoder, constructed by the convolutional layers and the ReLU layers without batch normalization layer. The autoencoder consists of an encoder and a decoder. The encoder pools the inputs into small size and high-dimension feature maps by nearest neighbor interpolation. Using bilinear interpolation, the decoder recovers feature maps back to images the same as the input. The reconstructed output of the autoencoder is supposed to as similar to the inputs as possible. The whole architecture of autoencoder is shown in Figure 5.

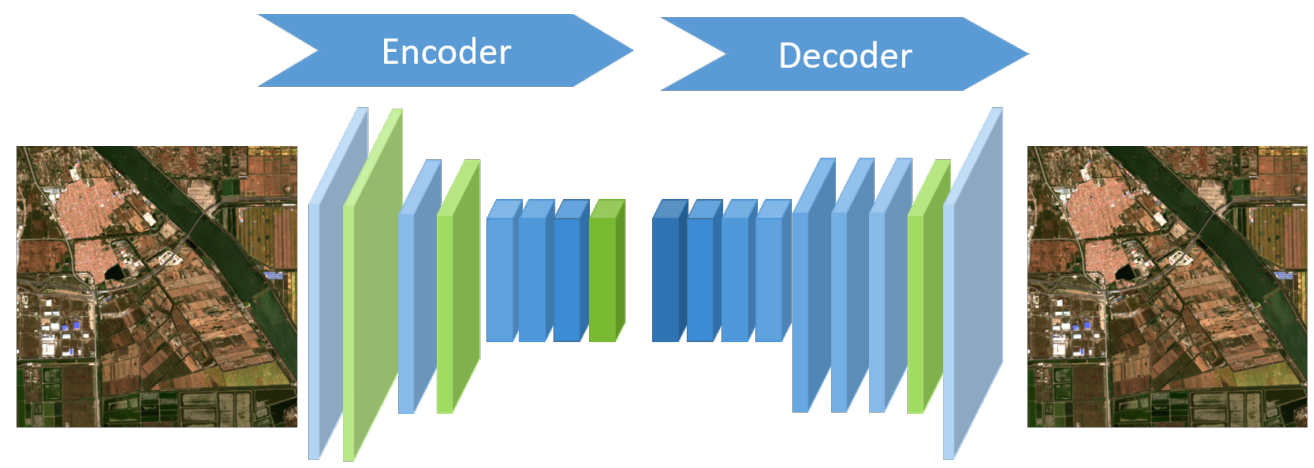

Figure 5. The architecture of our autoencoder for extracting feature maps. It consists of an encoder module and a decoder module. We mark some layers to green as the feature maps we adopt.

Though we replace the max-pooling layer, which partly discards location information, the autoencoder network still retains a pooling layer for thrift memory occupation. Therefore, we summarize features from varied layers to compose a perceptual loss hierarchically. Specifically, we select the features of layers 3, 8, 17, 34 in our autoencoder, which have been marked in green as perceptual features. This assures the proposed perceptual loss to contain both semantic and pixel-level information. As the variance of each feature map should describe the discrepancy between images as opposed to layers, we normalize the deviation of perceptual feature from each layer to 1 and sum their corresponding perceptual loss up.

As for the optimization of $\mathrm{D}$, we expect it to correctly distinguish between real and fake data. Furthermore, due to the diversity of samples, the weights of the $\mathrm{G}$ change significantly due to its high gradient, so we take the advantages of a gradient penalty [20] to avoid a complete change in one batch, and formed the discriminative loss as follows:

$$
\operatorname{Loss}_{D}=\text { Loss }_{\text {dist } \mid \text { fake }}-\text { Loss }_{\text {dist } \mid \text { real }}+\gamma\left(\sum g_{W_{i}}^{2}-1\right)^{2}
$$

where the Loss dist|fake refers to predicted 1-Lipschitz metrics $f_{\omega}$ when the sample is fake, and the Loss dist $\mid$ real refers to the otherwise situations. The last item refers to the gradient penalty, where the $\mathrm{g}_{W_{i}}$ refers to the gradient flow of each weight parameter for loss function. In summary, each image pair contributes gradient by generative and discriminative loss function aforementioned. However, considering patches merging issue, there should be a further batch inconsistency loss to improve performance, which is discussed in following sections. 


\subsection{Patches Clipping-And-Merging Method}

As deep learning networks can only accept small size images limited by memory, we crop images into patches to fit the network more often than not. To ensure the seam line between patches is natural and realistic, we crop patches with overlaps as most remote sensing deep learning applications do. The predicted SR patches should pose in their original area to compose the entire SR image, which brings diversity on how to address pixel value in overlaps. The high-level semantic tasks choose to take the average value of each patch. However, the average operation influences the clarity of images, against the purpose of improving image quality. On the other hand, as the overlap involves information from two patches, there is pixel value discontinuity between the overlapping and non-overlapping areas. These two phenomena get even worse when the overlaps of adjacent patches go more inconsistent, and get disappear as they come to the same. As long as the difference exists, roughly changing the overlapping rate or merging them in a weighted way cannot solve them both.

Thus, we design the clipping-and-merging method with the batch internal inconsistency loss to handle large-scale remote sensing images. First, as we found the patches inconsistency is source for image stitching problem, we encourage network to produce batch consistent results. We take $25 \%$ as the overlapping rate, as empirically we found it guides the two adjacent patches to getting similar receptive fields in the overlaps. Specifically, we crop the $168 \times 168$ sized images into $2 \times 2$ parts, namely, $96 \times 96$ sized patches, forming four overlaps of 24 pixels. We process these 4 patches as a batch into networks. Furthermore, we introduce the inconsistency loss, and thus the generative loss for this batch comes to

$$
\text { Loss }_{\text {batch }}=\text { Loss }_{\text {image }}+\delta \sum \text { Loss }_{\text {incons }}
$$

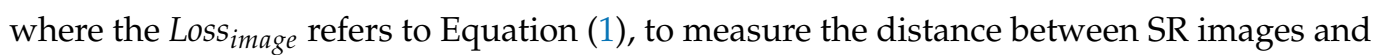
HR images. The inconsistency loss, Loss $_{\text {incons }}$, represents the L2 distance in each overlap between patches and is parameterized with $\delta$. This loss urges the network to predict similar results based on similar receptive fields as we designed.

To completely dismiss the risk of blurred phenomena from average operation, we adopt the clipping-and-merging method to predict large-scale remote sensing images. We crop the images into patches with overlaps as mentioned above, restore SR patches separately, and clip these patches before merging until no overlap left, as shown in Figure 6. Specifically, the out-half side of overlap in each cropped patch is clipped and discarded, while the inside and reliable half is retained. The overlap in the predicted result is composed from two neighbor patches half by half. Experiments show that images predicted by our method leave no visual seam line.

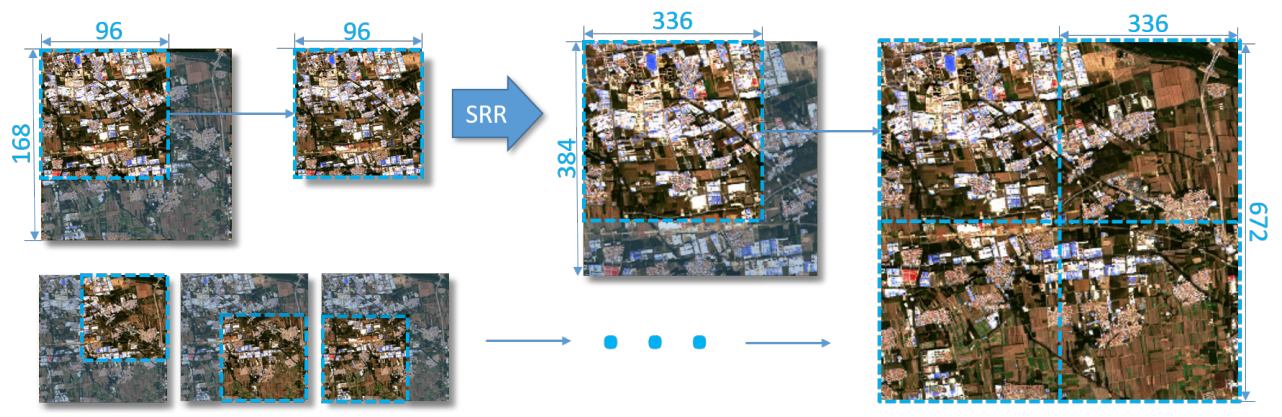

Figure 6. The pipeline of clipping-and-merging method. The input image is cropped into four patches with overlaps. Each patch is upsampled by 4 times through our networks, namely, $384 \times 384$ pixels. Half of overlap in each patch is clipped, and thus the patches get size of $336 \times 336$ pixels, namely a quarter of result. Therefore, four patches predicted by the aforementioned method compose the whole upsampling result. 


\section{Experiment}

Based on the structure mentioned above, we conduct experiments with the EnlightenGAN on mid-resolution remote sensing images. Concrete implementation details and experiment results are discussed in this section.

\subsection{Implement Details}

The experimental dataset is generated from Sentinel-2 images. Sentinel-2 is a midresolution imaging satellite carrying a multispectral imager for land monitoring. It can provide images of vegetation, soil and water cover, inland waterways, and coastal areas, as well as emergency rescue services. Sentinel-2 takes multispectral images at a height of $786 \mathrm{~km}$, covering 13 spectral bands and a width of $290 \mathrm{~km}$. We select bands 2, 3, and 4, representing blue, green, red bands, respectively, of 10-meter resolution to generate images for training and testing.

Thus, we train our model on two 10,980 × 10,980 size RGB images with rich texture and details information. These images are cropped into 423 images in size of $672 \times 672$ pixels. Among these images, we split them into 323 images for training and 100 images for testing. Those images are downsampled by 4 times to $168 \times 168$ pixels, and thus constitute the LR and HR image pairs. As mentioned before, we applied the cropping-and-clipping method to crop images into 4 patches with overlapping rate of 0.25 , namely, $96 \times 96$ pixels patches as the same with the input size of the $G$, and feed them into networks as a batch. When testing, we directly feed the $168 \times 168$ size images into network and attain the SR images, since the testing procedure costs less memory occupation than training does. Moreover, we took advantages of data augmentation operations online on the dataset for the generalizability of model, such as 90-degree rotation randomly several times.

Before training the Enlighten-GAN, we prepared the autoencoder in advance for the calculation of Self-Supervised Hierarchical Perceptual Loss. We trained the autoencoder for 200 epochs on the same dataset. The learning rate is set to $1 \times 10^{-4}$ as initial and get cosine-annealed during training.

For our proposed Enlighten-GAN, we trained the $G$ for 3 epochs with $\alpha, \lambda$ equal to 0 , namely, only content loss remaining, to initially enable the $G$ to produce reasonable images, forming a balance between the $\mathrm{G}$ and the $\mathrm{D}$ for a stable training procedure. Afterwards, we further train the $\mathrm{G}$ and the $\mathrm{D}$ with the $\alpha, \lambda$, and $\theta$ set to $0.001,0.006$, and 0.25 , respectively, in Equation (1); the $\gamma$ set to 10 in Equation (2); and the $\sigma$ set to 0.25 in Equation (3) for 200 epochs. We used Adam optimizer [32] for training with an initial learning rate of $1 \times 10^{-4}$. After 100 epochs, the learning rate is cut to 0.1 times of the previous value. The trained $G$ is used for mid-resolution remote sensing SRR task, and we only retain the 4-times upsampling results.

Our method was trained and tested all on the Supercomputing Center of Wuhan University with CPUs of Intel(R) Xeon(R) E5-2640 v4 and GPUs of Nvidia Tesla V100 16 GB. The autoencoder took $17 \mathrm{~h}$ to be trained, while the Enlighten-GAN took $15 \mathrm{~h}$.

\subsection{Image Quality Assessment}

Although visual quality has the final call, we still need a robust and reliable image quality assessment metric for weighing slight changes in the evaluation of SRR methods. Some previous works took Peak Signal to Noise Ratio (PSNR) as their metrics. After normalizing the images ranging from 0 to 1 , The PSNR is formed as

$$
P S N R=-10 \times \log (\text { MSE })
$$

where the MSE refers to mean square error between fake images and real images. However, PSNR-orient methods, such as pixel-loss-based methods, lead to smooth results as aforementioned. Intuitively, as shown in Figure 7, prediction with a little pixel geometry error leads to a lower PSNR, while a smooth map obtained a higher score. In ill-posed SRR methods, realistic portrait with inevitable geometry error is way meaningful than blurred outline, which implies the unreliability of PSNR. 


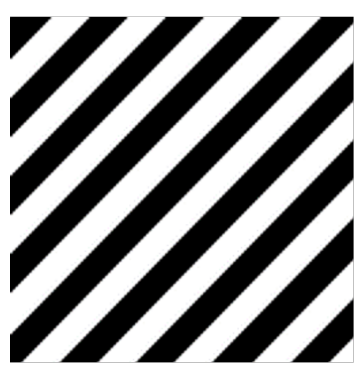

Ground Truth

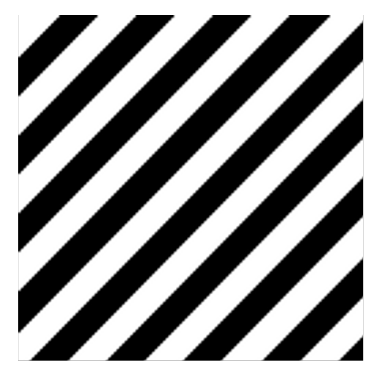

SR Image I

$(P S N R=0 / G S M=1.000)$

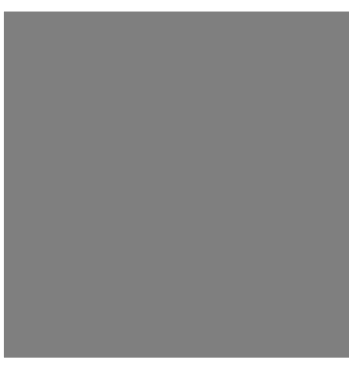

SR Image II

$(P S N R=6.02 / G S M=0.345)$

Figure 7. The flaws in PSNR. The second and third patches are the two SR results, while the first one is the ground truth. Notably, the second patch retain the basic shape, but due to the information loss, it introduces geometry error and swaps the white and black area when predicting, thus obtaining a lower PSNR than the third. The GSM metric, on the contrast, evaluates the results reasonably.

The others choose the Perceptual Index (PI), which is also the official metric of PIRMSR Challenge [33]. It is a mix-up of Ma's score [34] and Natural Image Quality Evaluator (NIQE) [35], which is respectively a pixel-level quality assessment and a non-reference perceptual assessment. It is formed as

$$
P I=0.5 \times((10-M a)+N I Q E)
$$

where a lower PI implies a richer texture. However, the pixel-level quality is in conflict with perceptual quality [36]. Thus, a lower PI metric does not necessarily depict higher pixel-level quality and perceptual quality at the same time. As a matter of fact, we found the results from ESRGAN with fatal artifacts attain a lower PI than ground truth in our experiment, demonstrated in the following subsection. Despite the lower PI implies richer texture, it is not assured to be real texture since the PI is a non-reference metric.

Therefore, we refer to related works and find the Gradient Similarity Metric (GSM) [37] has a better performance in sparse coding and reconstruction channel of TID2013 [38] in Zhang et al.'s experiment [39]. The TID2013 dataset synthesizes varied distortion images with certain ratio and evaluates metric with that ratio. The GSM weighs the correlation coefficients of gradient, defined as

$$
\operatorname{GSM}(x, y)=\frac{2 g_{x} g_{y}}{g_{x}^{2}+g_{y}^{2}}
$$

where the $g_{x}$ and $g_{y}$ refers to the gradient map of image $x$ and $y$.

To better prove the superiority of our method, we further introduce the Learned Perceptual Image Patch Similarity (LPIPS) to measure the perceptual difference between patches [40]. It is defined as

$$
d(x, y)=\sum_{l} \frac{1}{H_{l} W_{l}} \sum_{h, w}\left\|\omega_{l} \odot\left(\widehat{x}_{h w}^{l}-\widehat{y}_{h w}^{l}\right)\right\|_{2}^{2}
$$

where $\hat{x}^{l}$ and $\widehat{y}^{l}$ refer to the VGG features for layer $l$, and the $\omega_{l}$ refers the vector to scale the activations channel-wise. Thus, the LPIPS measures the perceptual distance between two patches with the VGG-Net. According the experiment conducted in [40], this metric performs well in the SRR task.

To assure fairness in our experiment, we still calculate the PSNR and PI of results from SRR methods besides the GSM. Notably, a lower LPIPS, a higher PSNR, and a higher GSM imply better performance. A low PI implies an image of a high information entropy. 


\subsection{Results of Evaluation}

We conducted evaluation experiment on our proposed method along with the input LR images and SR images from bicubic upsampling, SRCNN [5], SRGAN [1], ESRGAN [16], and EEGAN [18] methods. Thus, we obtained the result of all patches in metrics aforementioned for these SRR methods. We calculated the average and standard deviation from all patches for each method and list the quantitative results in Table 1. For better comparison, we also list the assessment of ground-truth as reference in the table. As the CNN-based method is trained on the pixel-loss equivalent to PSNR, and thus is more likely to gain high PSNR and oversmooth result, we suppose the best score at PSNR among GAN-based methods depicts the best result. The PI closest to ground truth suggests the results are of similar information entropy to the ground truth. Notably, we suggest the GSM is the most reliable metric among them, so it has the final call. As shown in Table 1, the Enlighten-GAN attains the best PSNR among GAN-based methods, the PI closest to ground truth, and the best GSM and LPIPS. Notably, our results get the lowest standard deviation in terms of GSM. We attribute it to our effort orienting to a stable model.

Table 1. The metrics of SR and HR images from varied method.

\begin{tabular}{ccccc}
\hline Method & GSM (avg/std) & LPIPS (avg/std) & PI (avg/std) & PSNR (avg/std) \\
\hline ground-truth & $1.000000 / 0.000000$ & $0.000 / 0.000$ & $2.493 / 0.501$ & $\infty /-$ \\
bicubic upsampling & $0.874414 / 0.031840$ & $0.557 / 0.065$ & $7.077 / 0.323$ & $23.881 / 2.737$ \\
SRCNN [5] & $0.949582 / 0.017110$ & $0.464 / 0.068$ & $6.736 / 0.534$ & $24.576 / 2.758$ \\
SRGAN [1] & $0.973279 / 0.007973$ & $0.293 / 0.038$ & $3.659 / 0.595$ & $19.413 / 2.812$ \\
ESRGAN [16] & $0.995795 / 0.002947$ & $0.189 / 0.027$ & $2.391 / 0.488$ & $22.368 / 2.744$ \\
EEGAN [18] & $0.998480 / 0.001953$ & $0.508 / 0.114$ & $3.439 / 0.707$ & $19.929 / 3.067$ \\
Enlighten-GAN & $0.999336 / 0.000750$ & $0.182 / 0.027$ & $2.509 / 0.546$ & $22.834 / 2.851$ \\
\hline
\end{tabular}

Qualitative results further depict our superiority to the other methods as shown in Figure 8. As we mentioned in the Section 1, the bicubic upsampling results and the SRCNN results are blurry, while the results of SRGAN are of stripped artifacts, depicted in every patch. Despite being designed for remote sensing images, the EEGAN is incompetent for mid-resolution remote sensing and produces spot artifacts. Among the state-of-the-art methods, the ESRGAN attains relative satisfying results, yet still suffers from the unstable convergence. The results from ESRGAN are of spot noise in the flattened area, such as lake and airport runway in the first and second rows. The hue of ESRGAN result does not follow the original LR patches and turns a little darker at the lake area as the second row demonstrate. ESRGAN tends to produce artifacts in the line with large color differences, such as the white line between yellow field and black road in the third row. As a contrast, the objects in our result basically retain their shapes and hues. The geometry offset in our SR images is far lower than the others, which implies the significant improvement from our methods.

Furthermore, to assure the effectiveness of proposed clipping-and-merging method, we crop our $168 \times 168$ images into four $96 \times 96$ patches, merge them into a whole in different method, and observe the overlap, as shown in Figure 9. The result from the clipping-and-merging method is merged without average operation, and thus is as sharp as the original patches. The common area of patches ensures the possibility for networks to predict the same outputs for adjacent patches. Therefore, the results are realistic in the overlaps. As a contrast, the result produced by averaging the two ESRGAN output patches contains an obvious seam line, cutting the building outline. In addition, the right side of the patches is blurred for average operation. 

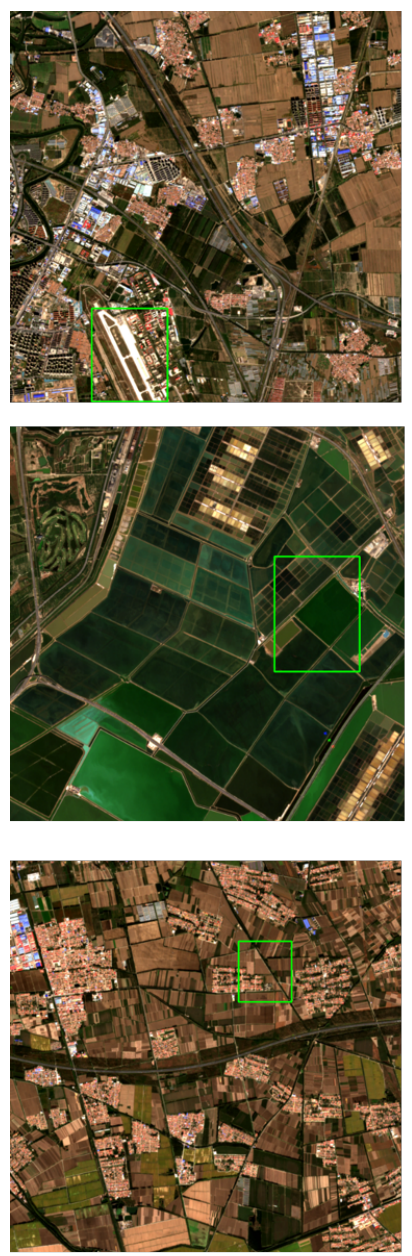

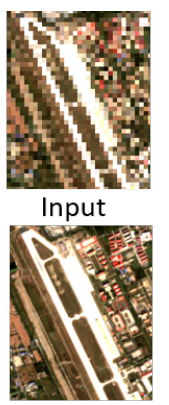

GT

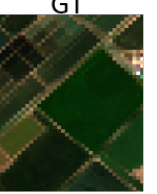

Input

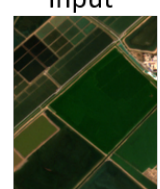

GT

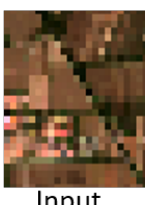

Input

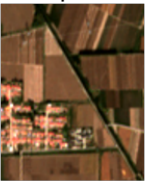

GT

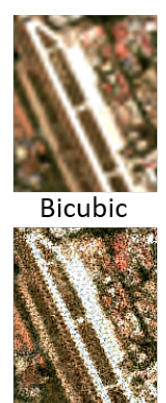

EEGAN

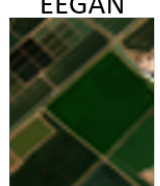

Bicubic

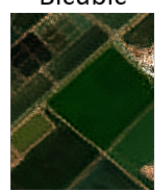

EEGAN

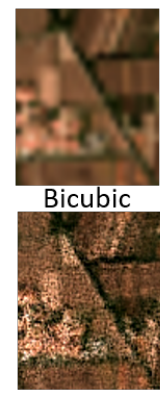

EEGAN

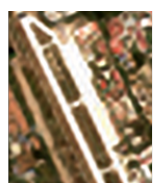

SRCNN
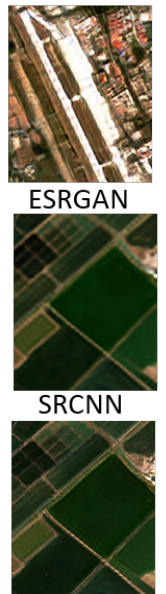

ESRGAN
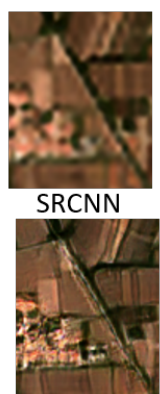

ESRGAN

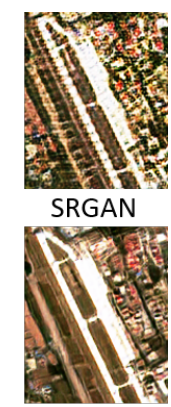

Ours

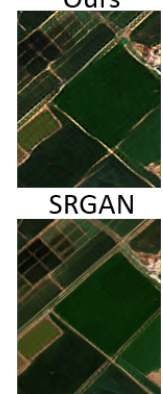

Ours

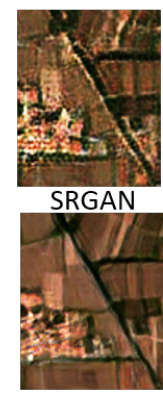

Ours

Figure 8. The quality result. We conduct experiment on varied methods. Our proposed Enlighten GAN outperforms the others.
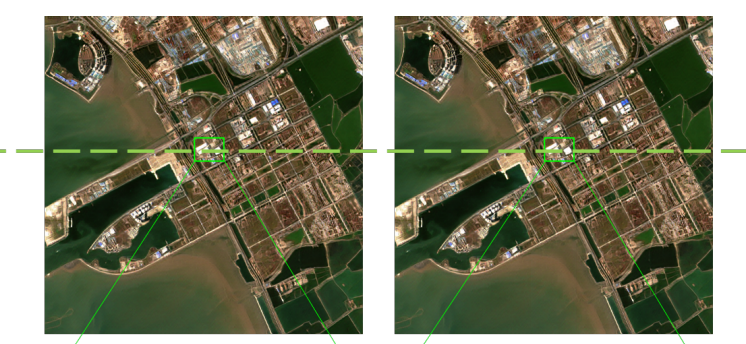

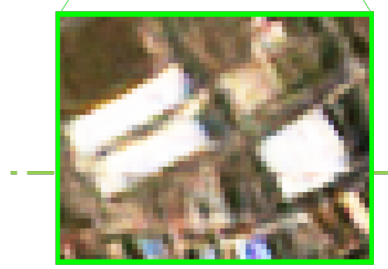

Average Merging

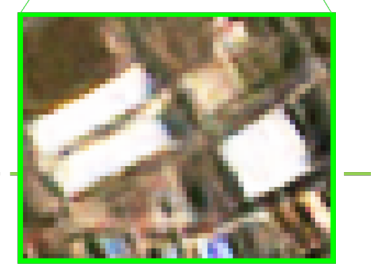

Clipping-and-Merging

Figure 9. The merged image and the detail information. The left one is average merging result from ESRGAN output, and the right one is the result from clipping-and-merging method. Two green dashed lines refer to the position where they merge. One can hardly find the seam line between patches without supplement of dashed lines in our method, while there is a clear line in the other one. 


\subsection{Ablation Study}

To illustrate the validation of our modification and support the views we mentioned above, we list results from some of our ablation study experiments. As the slight changes may not cause obvious affect in visual, we utilize the GSM along with the PI and PSNR to carefully compare varied tricks. The overall results are listed below.

Self-Supervised Hierarchical Perceptual Loss. We found setting $\lambda$ to zero in Equation (1) does not necessarily decrease the performance in ESRGAN. Therefore, we conduct an experiment to verify the effectiveness of our proposed Self-Supervised Hierarchical Perceptual Loss. We compared the model trained, respectively, with the proposed Self-Supervised Hierarchical Perceptual Loss, conventional VGGNet-based perceptual loss, and no perceptual loss. The results are shown in the Table 2, the second row list the assessments of our results.

Table 2. The comparison of varied perceptual loss.

\begin{tabular}{ccccc}
\hline Perceptual Loss & GSM (avg/std) & LPIPS (avg/std) & PI (avg/std) & PSNR (avg/std) \\
\hline ground-truth & $1.000000 / 0.000000$ & $0.000 / 0.000$ & $2.493 / 0.501$ & $\infty /-$ \\
our Perceptual & $0.999336 / 0.000750$ & $0.182 / 0.027$ & $2.509 / 0.546$ & $22.834 / 2.851$ \\
without Perceptual & $0.999204 / 0.001265$ & $0.228 / 0.028$ & $2.611 / 0.491$ & $22.291 / 2.655$ \\
VGG-Perceptual & $0.999112 / 0.000834$ & $0.169 / 0.027$ & $2.396 / 0.571$ & $22.485 / 2.850$ \\
\hline
\end{tabular}

VGG-Perceptual attains the best LPIPS, since they both designed on VGG-Net. However, in terms of GSM, the most reliable metric we propose, it is defeated by the model trained with no perceptual loss. It stands for the uncertainty we posed in Section 2. By contrast, our results obtain the best GSM, confirming the superiority of the SelfSupervised Hierarchical Perceptual Loss. Due to considering more on low-level features than VGG-Perceptual loss does, ours receives a slight deterioration in PI, yet it is closer to the ground-truth.

Wasserstein GAN. The main issue we face is the unstable convergence procedure. We turn to varied GAN structures for a better performance. As there is a great number of variants of GAN, we test on WGAN [20] which has been proved to be effective and RaGAN [24] applied in ESRGAN along with the standard GAN as baseline, to determine the best choice of our method. As shown in the Table 3, we found the WGAN attains the most satisfying result among them, namely, the best GSM, the best PSNR, and a passable LPIPS, along with a PI close to ground-truth. Under comprehensive consideration, we apply the WGAN and its non-activation 1-Lipschitz metric in our method.

Table 3. The comparison of varied variants of GAN.

\begin{tabular}{ccccc}
\hline GAN & GSM (avg/std) & LPIPS (avg/std) & PI (avg/std) & PSNR (avg/std) \\
\hline ground-truth & $1.000000 / 0.000000$ & $0.000 / 0.000$ & $2.493 / 0.501$ & $\infty /-$ \\
WGAN & $0.999336 / 0.000750$ & $0.182 / 0.027$ & $2.509 / 0.546$ & $22.834 / 2.851$ \\
Standard GAN & $0.994932 / 0.003888$ & $0.174 / 0.025$ & $2.338 / 0.523$ & $22.760 / 2.709$ \\
RaGAN & $0.998035 / 0.003349$ & $0.237 / 0.031$ & $2.784 / 0.494$ & $21.758 / 2.616$ \\
\hline
\end{tabular}

\section{Discussion}

From what is demonstrated in our experiments, the pixel-loss-based methods such as SRCNN attain stable SR images but suffer from the blur issue. They attain robust results with limited performance improvement. By contrast, the GAN-based methods such as SRGAN and EEGAN obtain sharp results when processing simple scene with few objects. When it comes to complicated scenes, the shallow network structures are not capable of predicting sophisticated details. Furthermore, the GAN-based methods induce the generative model to an unstable convergence, and thus product varied fatal artifacts. The artifact issue from ESRGAN is relieved, proving the effectiveness of the 
strategies of intensifying the network structure and stabilizing converging procedure. As shown in Figure 8, the results of our proposed method, owing to the enlighten block and Wasserstein GAN structure, are free of artifacts and retain the correct shape and hue.

Nevertheless, there is still an obvious gap between our results and the ground-truth. Taking a closer view, the shape of objects becomes distorted and their gaps disappear, especially in the town or urban area. As shown in Figure 10, the buildings in the edge area of town still retain their basic shape, and their corners are reliable for later application. When it goes to the central area, the networks cannot figure out the outlines of each objects from the chaotic background, especially in the area marked by green circles. Thus, the impact of surroundings and the complicated urban detail limit the performance of SRR models without external prior knowledge, even when we train on a dataset fully considering urban samples. This can be a direction to further work on.

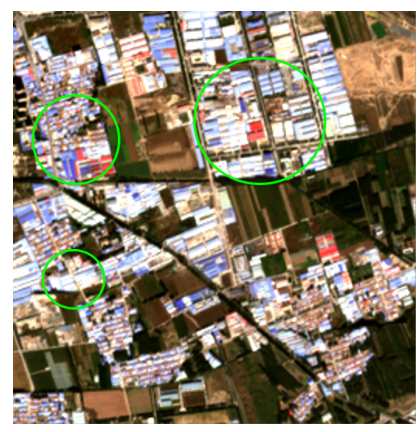

Ground Truth

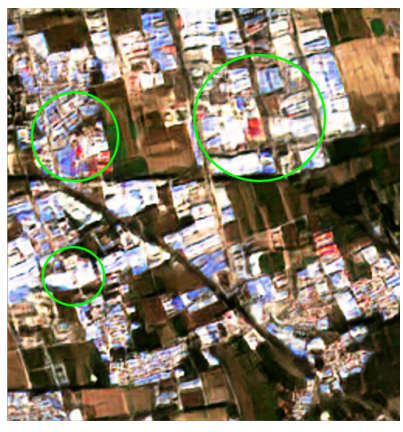

Our Results

Figure 10. The comparison of ground truth and SR images in the town scene. The remote sensing image in the town area is full of various objects with different colors. The networks cannot distinguish one building with the others in the complicate background.

\section{Conclusions}

We proposed an Enlighten-GAN method aiming at the mid-resolution optical remote sensing images SRR task. To overcome the unstable convergence, we exploit varied methods involving the enlighten block to guide the generating of feature maps, the SelfSupervised Hierarchical Perceptual Loss to optimize the Generative model, and the WGAN structure to stabilize the training process. Our experiments verify the superiority of our method. Moreover, we pioneer the merging problem for large remote sensing images. The results of images from our method turn to be realistic.

However, we found there is still a clear margin between our results and the groundtruth, especially in the urban area. The building objects are fused, and the outline of each building is unclear. In conclusion, the urban area in mid-resolution remote sensing images is an even more challenging issue to be solved in future.

Author Contributions: Conceptualization, Y.G. and L.Z.; Data curation, G.C.; Formal analysis, P.L.; Funding acquisition, X.Z. and G.C.; Investigation, Y.G.; Methodology, P.L.; Project administration, X.Z.; Resources, G.C.; Software, L.Z.; Supervision, X.Z.; Validation, P.L., L.Z. and G.C.; Visualization, P.L.; Writing — original draft, P.L.; Writing—review and editing, K.Z., X.T. and Z.L. All authors have read and agreed to the published version of the manuscript.

Funding: This work was supported by China Postdoctoral Science Foundation (No.2020M680109) and the Fundamental Research Funds for the Central Universities.

Institutional Review Board Statement: Not applicable.

Informed Consent Statement: Not applicable.

Data Availability Statement: All data, models, and code generated or used during the study will be available at GitHub soon. 
Acknowledgments: The authors would like to thank the developers of PyTorch and GDAL communities. The numerical calculations in this letter have been done on the supercomputing system in the Supercomputing Center of Wuhan University.

Conflicts of Interest: The authors declare no conflicts of interest.

\section{References}

1. Ledig, C.; Theis, L.; Huszár, F.; Caballero, J.; Cunningham, A.; Acosta, A.; Aitken, A.; Tejani, A.; Totz, J.; Wang, Z.; et al. Photo-realistic single image super-resolution using a generative adversarial network. In Proceedings of the IEEE Conference on Computer Vision and Pattern Recognition, Honolulu, HI, USA, 21-27 July 2017; pp. 4681-4690.

2. Stark, H.; Oskoui, P. High-resolution image recovery from image-plane arrays, using convex projections. J. Opt. Soc. Am. Opt. Image Sci. 1989, 6, 1715. [CrossRef] [PubMed]

3. Yang, J.; Wright, J.; Huang, T.; Ma, Y. Image super-resolution as sparse representation of raw image patches. In Proceedings of the 2008 IEEE Conference on Computer Vision and Pattern Recognition, Anchorage, AK, USA, 23-28 June 2008; pp. 1-8.

4. Yang, J.; Wright, J.; Huang, T.S.; Ma, Y. Image super-resolution via sparse representation. IEEE Trans. Image Process. 2010, 19, 2861-2873. [CrossRef] [PubMed]

5. Dong, C.; Loy, C.C.; He, K.; Tang, X. Learning a deep convolutional network for image super-resolution. In European Conference on Computer Vision; Springer: Berlin/Heidelberg, Germany, 2014; pp. 184-199.

6. He, K.; Zhang, X.; Ren, S.; Sun, J. Deep residual learning for image recognition. In Proceedings of the IEEE Conference on Computer Vision and Pattern Recognition, Las Vegas, NV, USA, 27-30 June 2016; pp. 770-778.

7. Kim, J.; Kwon Lee, J.; Mu Lee, K. Accurate image super-resolution using very deep convolutional networks. In Proceedings of the IEEE Conference on Computer Vision and Pattern Recognition, Las Vegas, NV, USA, 27-30 June 2016; pp. 1646-1654.

8. Zhang, Y.; Li, K.; Li, K.; Wang, L.; Zhong, B.; Fu, Y. Image super-resolution using very deep residual channel attention networks. In Proceedings of the European Conference on Computer Vision (ECCV), Munich, Germany, 8-14 September 2018; pp. $286-301$.

9. Tai, Y.; Yang, J.; Liu, X. Image super-resolution via deep recursive residual network. In Proceedings of the IEEE Conference on Computer Vision and Pattern Recognition, Honolulu, HI, USA, 21-26 July 2017; pp. 3147-3155.

10. Huang, G.; Liu, Z.; Van Der Maaten, L.; Weinberger, K.Q. Densely connected convolutional networks. In Proceedings of the IEEE Conference on Computer Vision and Pattern Recognition, Honolulu, HI, USA, 21-26 July 2017; pp. 4700-4708.

11. Tong, T.; Li, G.; Liu, X.; Gao, Q. Image super-resolution using dense skip connections. In Proceedings of the IEEE International Conference on Computer Vision, Venice, Italy, 22-29 October 2017; pp. 4799-4807.

12. Hu, J.; Shen, L.; Sun, G. Squeeze-and-excitation networks. In Proceedings of the IEEE Conference on Computer Vision and Pattern Recognition, Salt Lake City, UT, USA, 18-22 June 2018; pp. 7132-7141.

13. Johnson, J.; Alahi, A.; Fei-Fei, L. Perceptual losses for real-time style transfer and super-resolution. In European Conference on Computer Vision; Springer: Berlin/Heidelberg, Germany, 2016; pp. 694-711.

14. Sajjadi, M.S.; Scholkopf, B.; Hirsch, M. Enhancenet: Single image super-resolution through automated texture synthesis. In Proceedings of the IEEE International Conference on Computer Vision, Venice, Italy, 22-29 October 2017; pp. 4491-4500.

15. Simonyan, K.; Zisserman, A. Very deep convolutional networks for large-scale image recognition. arXiv 2014, arXiv:1409.1556.

16. Wang, X.; Yu, K.; Wu, S.; Gu, J.; Liu, Y.; Dong, C.; Qiao, Y.; Change Loy, C. Esrgan: Enhanced super-resolution generative adversarial networks. In Proceedings of the European Conference on Computer Vision (ECCV), Munich, Germany, 8-14 September 2018.

17. Lei, S.; Shi, Z.; Zou, Z. Super-resolution for remote sensing images via local-global combined network. IEEE Geosci. Remote. Sens. Lett. 2017, 14, 1243-1247. [CrossRef]

18. Jiang, K.; Wang, Z.; Yi, P.; Wang, G.; Lu, T.; Jiang, J. Edge-enhanced GAN for remote sensing image superresolution. IEEE Trans. Geosci. Remote Sens. 2019, 57, 5799-5812. [CrossRef]

19. Goodfellow, I.; Pouget-Abadie, J.; Mirza, M.; Xu, B.; Warde-Farley, D.; Ozair, S.; Courville, A.; Bengio, Y. Generative adversarial nets. Adv. Neural Inf. Process. Syst. 2014, 27, 2672-2680.

20. Arjovsky, M.; Chintala, S.; Bottou, L. Wasserstein gan. arXiv 2017, arXiv:1701.07875.

21. Mirza, M.; Osindero, S. Conditional generative adversarial nets. arXiv 2014, arXiv:1411.1784.

22. Nowozin, S.; Cseke, B.; Tomioka, R. f-gan: Training generative neural samplers using variational divergence minimization. In Proceedings of the 30th International Conference on Neural Information Processing Systems; Curran ssociates Inc.: Red Hook, NY, USA, 2016; pp. 271-279.

23. Miyato, T.; Kataoka, T.; Koyama, M.; Yoshida, Y. Spectral normalization for generative adversarial networks. arXiv 2018, arXiv:1802.05957.

24. Jolicoeur-Martineau, A. The relativistic discriminator: A key element missing from standard GAN. arXiv 2018, arXiv:1807.00734.

25. Dong, C.; Loy, C.C.; Tang, X. Accelerating the super-resolution convolutional neural network. In European Conference on Computer Vision; Springer: Berlin/Heidelberg, Germany, 2016; pp. 391-407.

26. Lim, B.; Son, S.; Kim, H.; Nah, S.; Mu Lee, K. Enhanced deep residual networks for single image super-resolution. In Proceedings of the IEEE Conference on Computer Vision and Pattern Recognition Workshops, Honolulu, HI, USA, 21-26 July 2017; pp. 136-144. 
27. Lai, W.S.; Huang, J.B.; Ahuja, N.; Yang, M.H. Deep laplacian pyramid networks for fast and accurate super-resolution. In Proceedings of the IEEE Conference on Computer Vision and Pattern Recognition, Honolulu, HI, USA, 21-26 July 2017; pp. 624-632.

28. Shocher, A.; Cohen, N.; Irani, M. “zero-shot" super-resolution using deep internal learning. In Proceedings of the IEEE Conference on Computer Vision and Pattern Recognition, Salt Lake City, UT, USA, 18-23 June 2018; pp. 3118-3126.

29. Jiang, K.; Wang, Z.; Yi, P.; Jiang, J.; Xiao, J.; Yao, Y. Deep distillation recursive network for remote sensing imagery super-resolution. Remote Sens. 2018, 10, 1700. [CrossRef]

30. Deng, J.; Dong, W.; Socher, R.; Li, L.J.; Li, K.; Fei-Fei, L. Imagenet: A large-scale hierarchical image database. In Proceedings of the 2009 IEEE Conference on Computer Vision and Pattern Recognition, Miami, FL, USA, 20-25 June 2009; pp. $248-255$.

31. Hinton, G.E.; Salakhutdinov, R.R. Reducing the dimensionality of data with neural networks. Science 2006, 313, 504-507. [CrossRef] [PubMed]

32. Kingma, D.P.; Ba, J. Adam: A method for stochastic optimization. arXiv 2014, arXiv:1412.6980.

33. Blau, Y.; Mechrez R.; Timofte R.; Michaeli T.; Zelnik-Manor L. The 2018 PIRM Challenge on Perceptual Image Super-Resolution In Computer Vision-ECCV 2018 Workshops. ECCV 2018; Lecture Notes in Computer Science; Leal-Taixé, L., Roth, S., Eds.; Springer: Cham, Switzerland, 2018; Volume 11133.

34. Ma, C.; Yang, C.Y.; Yang, X.; Yang, M.H. Learning a no-reference quality metric for single-image super-resolution. Comput. Vis. Image Underst. 2017, 158, 1-16. [CrossRef]

35. Mittal, A.; Soundararajan, R.; Bovik, A.C. Making a "completely blind" image quality analyzer. IEEE Signal Process. Lett. 2012, 20, 209-212. [CrossRef]

36. Blau, Y.; Michaeli, T. The perception-distortion tradeoff. In Proceedings of the IEEE Conference on Computer Vision and Pattern Recognition, Salt Lake City, UT, USA, 18-22 June 2018; pp. 6228-6237.

37. Liu, A.; Lin, W.; Narwaria, M. Image quality assessment based on gradient similarity. IEEE Trans. Image Process. 2011, 21, 1500-1512. [PubMed]

38. Ponomarenko, N.; Jin, L.; Ieremeiev, O.; Lukin, V.; Egiazarian, K.; Astola, J.; Vozel, B.; Chehdi, K.; Carli, M.; Battisti, F.; et al. Image database TID2013: Peculiarities, results and perspectives. Signal Process. Image Commun. 2015, 30, 57-77. [CrossRef]

39. Zhang, L.; Shen, Y.; Li, H. VSI: A visual saliency-induced index for perceptual image quality assessment. IEEE Trans. Image Process. 2014, 23, 4270-4281. [CrossRef] [PubMed]

40. Zhang, R.; Isola, P.; Efros, A.A.; Shechtman, E.; Wang, O. The unreasonable effectiveness of deep features as a perceptual metric. In Proceedings of the IEEE Conference on Computer Vision and Pattern Recognition, Salt Lake City, Utah, USA, 18-22 June 2018; pp. 586-595. 